\title{
ORIGINAL
}

\section{PREVALENCIA DE CONSUMO DE TABACO ENTRE LAS MÉDICAS Y LAS ENFERMERAS DE LA COMUNIDAD DE MADRID (*)}

\author{
M. ${ }^{a}$ Luisa Fernández Ruiz (1) y Marciano Sánchez Bayle (2) \\ (1) Fundación para la Investigación, Estudio y Desarrollo de la Salud Publica \\ (2) Grupo de Trabajo sobre Factores de Riesgo Cardiovascular. Hospital del Niño Jesús. \\ $\left(^{*}\right)$ Trabajo realizado con una ayuda de la Dirección General de la Mujer. Consejería de Sanidad y Servicios So- \\ ciales. Comunidad de Madrid.
}

\section{RESUMEN}

Fundamentos: El tabaquismo es responsable de una elevada mortalidad y morbilidad en nuestro país. El tabaquismo de los profesionales de la salud tiene intluencia sobre los hábitos de la población. En España, el tabaquismo esta incrementándose entre las mujeres. El objetivo de nuestro estudio es estudiar la prevalencia de consumo de tabaco entre las médicas y enfermeras de la Comunidad de Madrid, así como sus actitudes y conocimientos respecto al tabaquismo.

Métodos: Se ha realizado un estudio descriptivo transversal entre las mujeres profesionales de la salud (médicas y enfermeras) de la Comunidad de Madrid, mediante una encuesta sobre hábito tabáquico, en un muestreo polietápico por conglomerados.

Resultados: Se han estudiado 1.235 profesionales ( 435 médicas y 800 enfermeras). El 43,07\% del total son fumadoras, siendo el tabaquismo mayor en las enfermeras $(47,62 \%)$ que en las médicas $(34,71 \%)$, y entre quienes trabajan en atención especializada $(46,68 \%)$ que entre las que lo hacen en atención primaria $(35,29 \%)$. El tabaquismo fue diferente en ambas profesiones según la edad En las médicas era menor el tabaquismo en las edades entre 20 y 30 años $(22,88 \%)$, y en las enfermeras este grupo de edad era el que tenia un mayor porcentaje de fumadoras $(52.38 \%)$. Eran exfumadoras el $18.3 \%$ del total de las encuestadas, y el $64,65 \%$ de las que fumaban señalaron que habian intentado dejarlo. Un 2,93\% fumaban delante de los/as pacientes, y un $14,94 \%$ pensaban que debia permitirse fumar en las salas de espera. El 27,46\% creían que estaba permitido fumar en su area de trabajo, y el $90,64 \%$ señaló que se fumaba labitualmente en las salas de uso común del personal sanitario, y un $30.37 \%$ respondió que cxistía un lugar cspccifico para fumar cn cl centro de trabajo.

Conclusiones: La prevalencia de tabaquismo entre las profesionales de la salud, médicas y enfermeras, de la Comunidad de Madrid, es elevada, superior a la de la población general de igual edad y genero. El tabaquismo entre las enfermeras es más frecuente que entre las médicas, y en los hospitales que en Atención Primaria, y entre las enfermeras en el grupo más joven, lo que es muy preocupante. El cumplimiento de la legislación sobre tabaco en los centros sanitarios parece muy bajo.

Palabras clave: Tabaquismo. Enfermeras. Médicas. Personal sanitario.

Correspondencia:

M. ${ }^{a}$ Luisa Fernández Ruiz

Fundación para la Investigación, Estudio

y Desarrollo de la Salud Publica.

c/ Loeches, 6,Bajo D

28008. Madrid.

\section{ABSTRACT
Smoking Prevalence of Women Physicians and Nurses in the Community of Madrid}

Background: Tobacco is a major causc of morbility and mortality in our country. The tobacco smoking by the sanitary staff has a negative influence in the rest of the population. In Spain women smoke more than men. The subject of our study is to identify the tobacco consume in the female sanitary staff in the Community of Madrid.

Methods: Transversal descriptive study in the female sanitary staff in the Community of Madrid, using a conglomerated polietapic sampling.

Results: We studied 1,235 professionals (435 medicine doctors and 800 nurses). $43.07 \%$ of them were smokers, with the higher rate in nurses $(47,68 \%)$ that are working at hospitals $(46.68 \%)$. On one hand the tobacco consumption was smaller in medicine doctors between $20-30$ years old $(22.88 \%$ ). On the other hand it turned out that nurses in their twenties formed a large group of smokers. A $18.3 \%$ of the total sample were actually smokers and a $64.65 \%$ had tried to give it up. A $2.93 \%$ of woman smoked without taking care if patients was close and $14,94 \%$ thought that it had to be allowed to smoke in the hospitals halls. $27.46 \%$ of smokers thought that smoking was allowed in their hospitals and $90,84 \%$ said that they usually smoked in the sanitary staff rooms, and $30.37 \%$ of them answered that specific rooms for smokers were available in their working places.

Conclusions: The prevalence of smoking in the health professionals in the Community of Madrid is very high. Medicine doctors smoke more than nurses, but young nurses smoke than young medicine doctors. Sanitary staff do not obey the no-smoking law in hospitals. The observance of this law is very low.

Key words: Smoking habit. Nurses. Female physicians. 


\section{INTRODUCCIÓN}

El tabaquismo, es un problema de salud pública de primera magnitud en nuestro país. Es la primera causa aislada de mortalidad y morbilidad en cáncer de pulmón y enfermedades cardiovasculares, y se calcula que es el responsable de unas 50.000 muertes anuales en España ${ }^{1}$. Los/as profesionales de la salud tienen un papel especialmente relevante en cuanto al tabaquismo de la población general, porque pueden influir en el mismo de cuatro maneras: Sirviendo como ejemplos o modelos; actuando como educadores sanitarios; promoviendo la aplicación de la legislación sobre consumo de tabaco en los centros sanitarios; y sensibilizando a la sociedad sobre los problemas derivados del tabaquismo ${ }^{2}$.

Se ha demostrado que el papel de los/as profesionales es muy importante a la hora del cambio social respecto al tabaquismo, y que el propio hábito tabáquico de los/as profesionales tiene una influencia relevante en cuanto a sus actitudes al respecto ${ }^{3,4}$.

Las diferentes encuestas que se han realizado entre profesionales de la salud en España ${ }^{1-14}$ revelan que, aparte de que la prevalencia de tabaquismo es mayor entre los/as profesionales de la salud que entre la población general, además se está produciendo, como también sucede entre la población general, un aumento del consumo entre las mujeres y una disminución entre los hombres, lo que hace que en las edades más jóvenes predominen las fumadoras ${ }^{15}$. Por ello, el objetivo de nuestro trabajo ha sido medir la prevalencia de tabaquismo entre las profesionales sanitarias, médicas y de enfermería, de la Comunidad de Madrid.

\section{MATERIAL Y MÉTODOS}

Se ha realizado un trabajo descriptivo transversal de las profesionales de la salud (médicas y enfermeras) de la Comunidad de Madrid, utilizando una encuesta con un cuestionario estructurado y cerrado (Anexo I), administrado personalmente por encuestadores/as entrenados en el propio centro de trabajo, el cual ya había sido empleado en otros estudios sobre tabaquismo en profesionales de la salud en la Comunidad de Madrid, con la intención de que sirviera para realizar comparaciones con estudios anteriores ${ }^{6}$.

Se ha definido como base muestral a las médicas y enfermeras que trabajan en la $\mathrm{Co}-$ munidad de Madrid, utilizando los directorios de los centros públicos y privados. Se buscó esta base muestral (trabajadoras en activo) porque son el grupo de las profesionales de la salud que tiene mayor interés, debido a su capacidad real para intervenir sobre los hábitos de la población. Se definió una muestra de 435 médicas y 800 enfermeras con un error teórico de $\pm 3,78 \%$ para un intervalo de confianza del $95 \%$, con $\mathrm{p}=\mathrm{q}$, para una pregunta dicotómica considerada como variable principal (la prevalencia de tabaquismo).

Se realizó un muestreo polietápico por conglomerados, basándose en el puesto de trabajo, seleccionado por centro de trabajo, $\mathrm{y}$ en estos por servicios y/o unidades (en el ámbito hospitalario).

Con el fin de incluir también a las profesionales más jóvenes de enfermería en la muestra, se comenzó la realización de la encuesta en agosto (momento en el que debido a las vacaciones del personal fijo hay un elevado número de suplencias), y se finalizo en noviembre de 1998.

En el caso de las profesionales de enfermería, y dado que las mujeres son el $83,3 \%$ del total de los enfermeros/as colegiados/as, los resultados se acercan a los que se obtendría en el conjunto de la profesión.

Se han realizado comparaciones entre porcentajes utilizando la chi cuadrado. 


\section{RESULTADOS}

Se han estudiado un total de 1.235 profesionales médicas y de enfermería de la Comunidad de Madrid, de ellas 435 eran médicas y 800 enfermeras. Del total de las personas estudiadas 391 trabajan en Atención Primaria, y el resto en Atención Especializa- da, de éstas últimas 721 los hacen en hospitales y 123 en ambulatorios de especialidades.

Se buscó una representación similar de todas las Areas de Salud en las que está distribuida la Comunidad de Madrid. La figura 1 recoge el número de profesionales estudiadas de cada una de ellas.

Figura 1

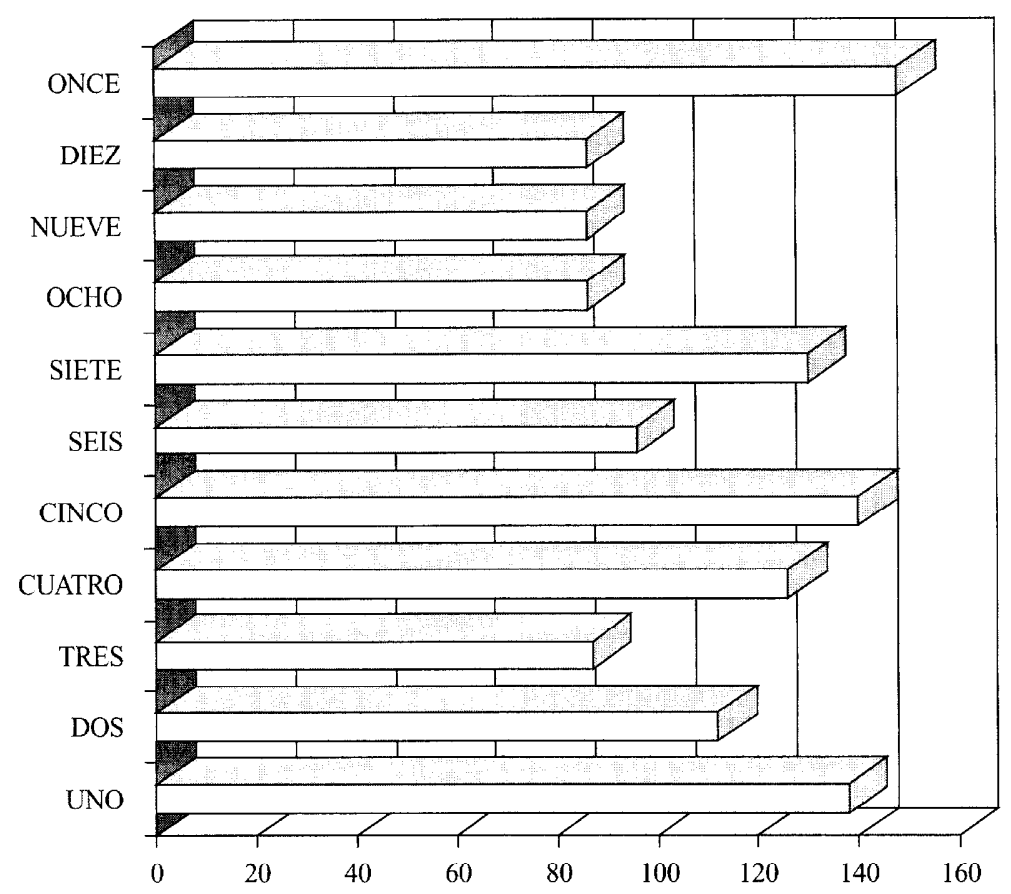

La media de edad del total de las personas estudiadas fue de 36,59 años (desviación estándar de 9,27 años), la edad de las médicas y las enfermeras fue similar (36,57 versus 36,59 respectivamente). En la figura 2 se observa la distribución de las encuestadas según edad y profesión.

Un total de $532(43,07 \%)$ de las mujeres encuestadas fumaban (IC 48,47-40,31\%). Un $8,42 \%$ del total eran fumadoras ocasionales (consumidoras de menos de 1 cigarrillo al día), y un $34,65 \%$ fumaban todos los días.
La prevalencia de tabaquismo fue diferente según la profesión: el 34,71\% (IC $39,18-30,24 \%$ ) de las médicas fumaban, frente al $47,62 \%$ de las enfermeras (IC 51,08-44,16\%).Esta diferencia era estadísticamente significativa para una $\mathrm{p}<0.001$.

La mayoría fuma predominantemente cigarrillos, sólo algunas (8 casos) señalan consumir algún otro producto (puros y/o tabaco de pipa). La media de cigarrillos que se consume diariamente es de 14,22 con una desviación estándar de 7,97 (rango de 1 a 60 ), la mayoría fumaba menos de 20 cigarri- 


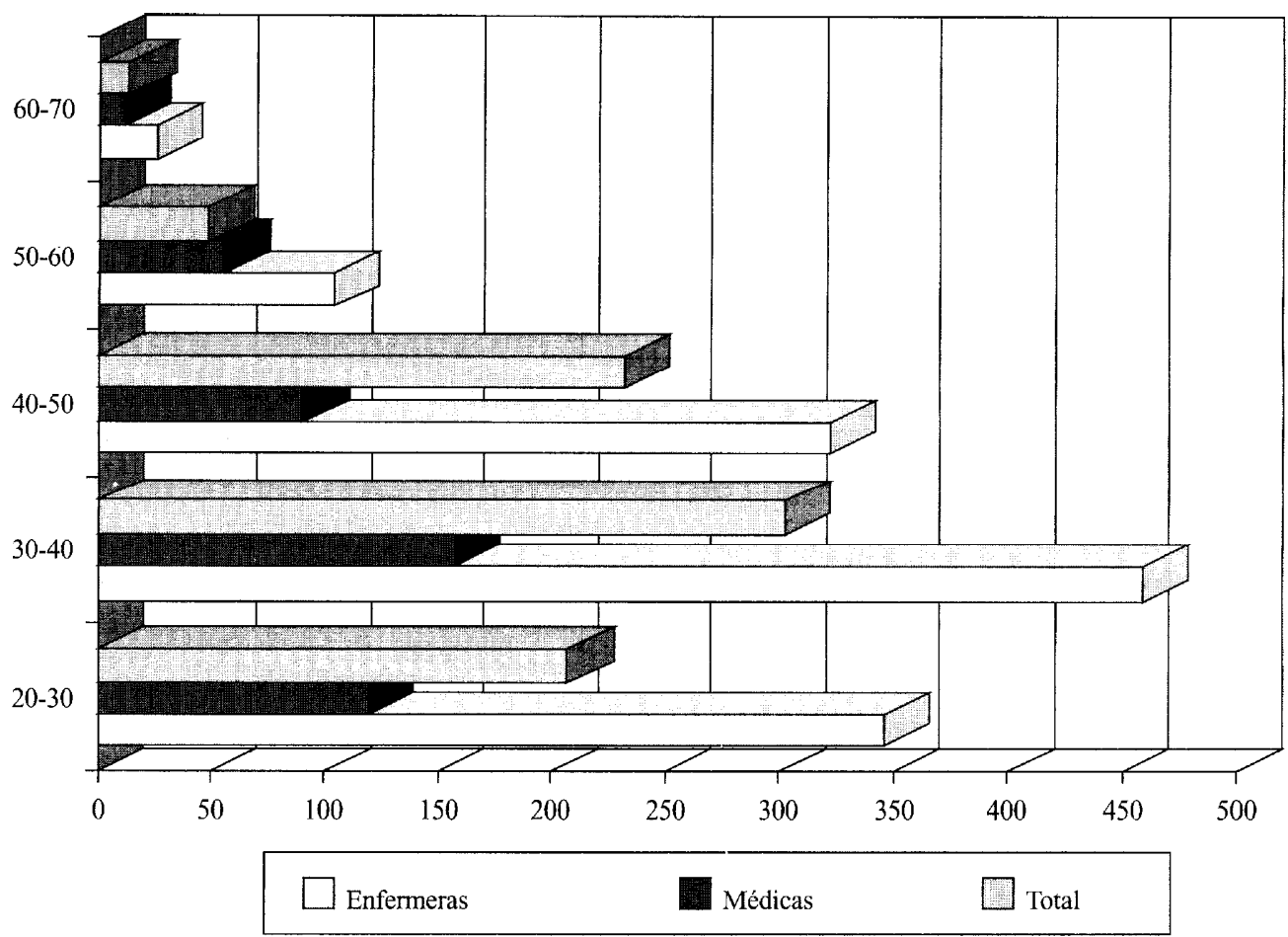

llos diarios (el 86,64\% del total: $90,26 \%$ de las médicas y $84,80 \%$ de las enfermeras).

La prevalencia de tabaquismo fue diferente según el lugar de trabajo. Fumaban el $35,29 \%$ (IC: 43,96 al 26,62\%) de las que trabajaban en Atención Primaria, y el 46,68\% (IC: $50,04-43,32 \%$ ) de quienes lo hacían en Atención Especializada (el 47,15\% de las que lo hacían en hospitales y el $43,9 \%$ de las que trabajaban en ambulatorios de especialidades). La diferencia entre ambos grupos (Primaria y Especializada tuvo significación estadística para una $\mathrm{p}=0.007$ ).

En la tabla 1 se recoge la prevalencia de tabaquismo según grupos de edad, tanto para médicas como para enfermeras. En ella se observa que la prevalencia de tabaquismo es diferente en las médicas que entre las profesionales de enfermería. En las médicas es menor en el grupo de menores de 30 años, y superior entre las edades de 30-40 años. En el caso de las enfermeras, la mayor prevalencia de tabaquismo se observa en el grupo más joven, y es menor en el grupo de más edad.

Tabla 1

Prevalencia de tabaquismo según edad y profesión

\begin{tabular}{|cccccc|}
\hline Edad (años) & \multicolumn{2}{c}{ Médicas } & \multicolumn{2}{c|}{ Enfermeras } \\
\hline $20-30$ & 27 & $22,88 \%$ & 110 & $52,38 \%$ \\
$30-40$ & 60 & $38,21 \%$ & 137 & $45,51 \%$ \\
$>40$ & 48 & $34,78 \%$ & 133 & $43,42 \%$ \\
\hline
\end{tabular}

El porcentaje de mujeres exfumadoras sobre el total de las encuestadas es del $18,83 \%$. Entre las que actualmente no fu- 
man, el $63,86 \%$ no lo habían hecho nunca y el $36,13 \%$ eran ex-fumadoras.

El $64,65 \%$ de quienes fuman señalan que han intentado dejar de fumar (el 73,91\% de las médicas, y el $61,56 \%$ de las enfermeras), siendo el porcentaje similar entre las mayores y menores de 40 años $(64,34 \%$ versus $65,34 \%)$.

Al preguntar a las fumadoras sobre cual piensan que será su consumo en el futuro, un $41,80 \%$ piensan que dentro de 5 años no fumaran y un $72,34 \%$ que habrán disminuido su consumo de tabaco.

Sobre el efecto nocivo del tabaco sobre los/as no fumadores, el $91,25 \%$ señalaban que el humo del tabaco perjudicaba a los/as no fumadores/as (lo que opinaban el $95,23 \%$ de las no fumadoras y el $89,76 \%$ de las fumadoras).

Respecto al conocimiento de la legislación vigente sobre tabaquismo el $60,14 \%$ del total referían conocerla $(61,66 \%$ de las no fumadoras y $60,05 \%$ de las fumadoras).

El 2,93 \% del total de las encuestadas fumaban en presencia de los/as pacientes.

Respecto a la permisión de fumar a los/as usuarios/as en la sala de espera, un $14,94 \%$ del total (IC: 16,92-12,96\%), encontrándose diferencias según el hábito tabáquico: $10,72 \%$ de las fumadoras y $19,84 \%$ de las no fumadoras pensaban que deberia permitirse fumar $(\mathrm{p}=0.002)$.

La valoración de los motivos para dejar de fumar está recogida en la tabla 2, siendo el principal el de proteger la salud, y el moti-

Tabla 2

Valoración positiva de los motivos para dejar de fumar en el total de las mujeres encuestadas

\begin{tabular}{|lr} 
Proteger su salud & $1.202(97,33 \%)$ \\
Aparición de sintomas & $1.077(87,20 \%)$ \\
Presión de compañeros/as & $590(47,77 \%)$ \\
No molestar & $1.038(84,05 \%)$ \\
Dar un buen ejemplo & $760(61,54 \%)$ \\
\hline
\end{tabular}

vo menos considerado la presión de los/as compañeros/as.

En cuestiones sobre aspectos relacionados con el tabaquismo en los centros sanitarios, consideraban que estaba permitido fumar en el area de trabajo el $27,46 \%$ de las encuestadas (IC: 29,94-24,98\%) con diferencias según el hábito tabáquico: el 39,84\% de las fumadoras frente al $16,12 \%$ de las no fumadoras $(\mathrm{p}<0.001)$. Un $90,64 \%$ (IC: $92,26-89,02 \%$ ) señala que se fuma habitualmente en las salas de uso común $(90,6 \%$ de las fumadoras y $91,96 \%$ de las no fumadoras) y solo un $30,37 \%$ dice que en su area de trabajo existe un lugar especifico para los/as fumadores/as $(36,04 \%$ de las fumadoras versus $25,35 \%$ de las no fumadoras, $\mathrm{p}=0.007$ )

\section{DISCUSIÓN}

El tabaquismo es un problema de primer orden de salud pública en los países desarrollados ${ }^{16,17}$. En España el tabaquismo en la población es elevado ${ }^{1}$, y también en la Comunidad de Madrid ${ }^{18}$. Se esta produciendo un cambio en el hábito tabáquico según el genero. El tabaquismo esta disminuyendo entre los hombres, que eran el grupo más fumador y, sin embargo, esta aumentando en las mujeres, especialmente entre las mas jóvenes. En 1997 en la Comunidad de Madrid fumaban el $53,1 \%$ de las mujeres entre $18 \mathrm{y}$ 30 años, y el 44,9\% en el grupo de edad entre los 30 y los 45 años ${ }^{18}$, esta situación parece corresponderse bastante bien con los datos obtenidos en nuestra encuesta entre las profesionales de enfermería.

En cuanto a las profesionales médicas hay varios estudios, tanto de ámbito estatal como de Comunidades Autónomas, entre ellas de la Comunidad de Madrid, con los que establecer comparaciones.

En los de ámbito estatal la prevalencia es más elevada que en nuestro caso $(52,6 \%$ SEPAR 1985 y 50,5\% Estudio HTA- Taba- 
co 1995) ${ }^{1}$. En los de Cataluña se ha observado una disminución del tabaquismo en los estudios de los últimos 14 años $(43,6 \%$ en 1982 frente a $31,4 \%$ en 1996) ${ }^{1,5}$. En la Comunidad de Madrid existe otro estudio en 1995 con una prevalencia ligeramente inferior $(31,6 \%)^{6}$.

Entre las profesionales de enfermería no hay estudios representativos de ámbito estatal, aunque las referencias señalan una prevalencia mayor de tabaquismo ${ }^{14}$. En Cataluña los datos publicados refieren una cierta tendencia a la disminución $(42,4 \%$ en 1985 y $40,6 \%$ en 1990). En otras Comunidades Autónomas las cifras son variables pero en márgenes elevados $(57,7 \%$ en los hospitales de Asturias en 1989,51,5\% en La Rioja en $1993,57,7 \%$ en el Hospital de Txagorritxu en 1997) ${ }^{7,8,12}$. En Madrid no existen datos a nivel de la Comunidad Autónoma, pero en 1996 en Atención Primaria del Area 1 se ha señalado una prevalencia del $42,62 \%{ }^{19}$, similar a la encontrada en nuestro caso.

A nivel internacional, muy especialmente en Europa, el tabaquismo entre las profesionales de la salud es netamente inferior al que se encuentra en España (en el Reino Unido la prevalencia es inferior al $10 \%)^{1}$.

Las actitudes respecto a algunos aspectos relacionados con el tabaco son distintos entre las fumadoras y no fumadoras, así sucede en cuanto al efecto del tabaquismo pasivo, claramente establecido por la literatura cientifica ${ }^{1} \mathrm{y}$ en cuanto a la permisividad de fumar en las salas de espera a los/as usuarios/as. Lo mismo sucede cuando se pregunta sobre si está permitido o no fumar en el puesto de trabajo, lo que afirman el $27,46 \%$ de las encuestadas $(39,84 \%$ de las fumadoras frente al $16,12 \%$ de las no fumadoras) a pesar de la legislación vigente al respecto ${ }^{20}$. Las fumadoras piensan más que las no fumadoras que existen lugares específicos para fumar, lo que no ha sido constatado por los/as encuestadores/as. Estas diferencias en las actitudes y percepciones, entre fumado- res/as y no fumadores/as, ya han sido referidas por otros autores ${ }^{6,10,11}$.

También llama la atención el reconocimiento por parte de casi todas las mujeres encuestadas $(90,64 \%)$, fumadoras o no, de que se fuma habitualmente en los centros sanitarios en los lugares de uso común.

Fl tabaquismo en las mujeres es además un problema especialmente preocupante por encontrarse en un momento emergente en la sociedad española, lo que ya ha sido señalado por otros autores ${ }^{1.15,21}$.

En conclusión, de nuestro estudio parece deducirse que la prevalencia de tabaquismo de las profesionales de salud de la Comunidad de Madrid (médicas y enfermeras) es elevada, superior a la de las mujeres de la población general. Asimismo, la prevalencia de tabaquismo entre las profesionales de enfermería es muy superior a la encontrada entre las medicas, por lo que éste colectivo debe de merecer una atención especial al respecto. El porcentaje de tabaquismo es mayor entre las profesionales de enfermería y entre las médicas de los hospitales respecto a las que trabajan en Atención Primaria.

Los grupos más jóvenes (menores de 30 años) de las médicas tienen una menor prevalencia de tabaquismo, lo que podría señalar una posible disminución del mismo entre estas profesionales en el futuro. En cambio, en el caso de las enfermeras, las mas jóvenes son las que fuman en mayor porcentaje, lo que es muy preocupante.

Las actitudes ante el tabaquismo son claramente distintas entre las fumadoras y las no fumadoras, condicionando el cumplimiento de las normas vigentes sobre tabaco.

Todo ello aconseja una intervención activa respecto a las profesionales de la salud de la Comunidad de Madrid para disminuir la prevalencia de tabaquismo, muy especialmente entre las profesionales de enfermcría. 


\section{BIBLIOGRAFÍA}

1. Comité Nacional para la Prevención del Tabaquismo: Libro blanco sobre el tabaquismo en España. Barcelona: Glosa; 1998.

2. Salvador LLivina T: Tabaquismo y responsabilidad profesional en el ámbito de la Salud Pública. Rev San Hig Publica 1990,64:585-588.

3. Abclin $\mathrm{T}$ :Working with professional groups to increase priorities for smoking education. En: Proceeding Third World Conference on Smoking and Health. Washington 1975. DHEN.

4. Russell MAH, Wilson C, Baker CD: Effect of general practitioners advice against smoking. BMJ 1979,2:231-235.

5. Salleras L1, Pardell H, Canela J, Vaque J, Plans P, Cobo E: tabac i professionals sanitaris. Salut $\mathrm{Ca}$ talunya 1987,1:69-74.

6. FADSP: Conocimientos y actitudes relacionados con el tabaco de los médicos del sector publico de la Comunidad de Madrid. Tabaco Hoy 1995,1:5-25.

7. Rubio Montaner L, Urueña Guzmán A, Santamaria Roa A, de la Hoz Olalla J, Machín Aguilera P, Acitores Augusto JM: Prevalencia del habito de fumar en los profesionales sanitarios de la Rioja. $\Lambda$ ten Primaria 1994,13:373-377.

8. Martinez Sande JL, Casariego Roson JR, Alonso Orcajo N, Rodríguez Llorian A: Estudio del tabaquismo en profesionales sanitarios del medio hospitalario de Asturias. Rev Esp Cardiol 1990,43:219-226.

9. Berraondo I, Antero E, Millet M, Saez M, Sampedro E: Consumo de tabaco entre los médicos de atención primaria de la Comunidad Autónoma del País Vasco. Aten Primaria 1994,14:838-840.
10. Sampedro Martínez E, Narzabal Goñi MA, Frias Oyaga O, Antero Berganzos E, Saez Salazar M, Aranegui Lasuen MC, Millet Sampedro M: Actitud ante el tabaco y prevalencia de fumadores en médicos de Atención Primaria de Guipúzcoa. Aten Primaria 1994,14:1073-1076.

11. López Perez P, García Sadaba I, Simarro Bravo MD, Blasco Cabrera L, Cebrian Valero T, Méndez Martínez C: Habito tabáquico y otros aspectos relacionados con el tabaco en los profesionales de Atención primaria de la provincia de Córdoba. Semergen 1996: 635-646.

12. Arevalo Alonso JM, Baquedano Arriazu FJ. Prevalencia del tabaquismo en los trabajadores de un hospital. Rev Esp Salud Publica, 1997,71:451-462.

13. Valero Alonso R, Sainz Saenz-Torre N, Torres Jimenez JI, Castanedo Saiz JA, Salido Cano A: Enfermeria y tabaquismo. Rol Enfermería 1988,122:17-19.

14. Heras Tebar A: Tabaquismo en enfermería. Rol de Enfermería 1997;232:57-60.

15. Chollat-Traquet $\mathrm{C}$ : La mujer y el tabaco. Ginebra: OMS; 1993.

16. Bartechi CE, Mc Kenzie TD, Schrier RW: The human costs of tobacco use (First of two parts).N Engl J Med;1994,330:907-912.

17. Bartechi CE, Mc Kenzie TD, Schrier RW: The human costs of tobacco use (Second of two parts).N Engl J Med, 1994;330:975-980.

18. Vigilancia sobre el consumo de tabaco y sus efectos para la salud en la Comunidad de Madrid. Bol Epidemiol Comunidad de Madrid 1997;11:3-27

19. Fernández Ruiz ML, Sánchez Bayle M: Hábitos, actitudes y conocimientos de los profesionales de enfermería en atención primaria respecto al tabaco. Centro de Salud 1997,5:186-191.

20. RD 510/1992. B.O.E. 14/5/1992

21. Jimenez Ruiz CA: Tabaco y mujer. Arch Bronconcumol 1996, 32:38-46. 


\section{ANEXO 1}

\section{CUESTIONARIO SOBRE CONSUMO Y ACTITUDES RESPECTO AL TABACO DE LAS PROFESIONALES SANITARIAS}

1. Edad (años):

2. ¿Cuantos años lleva trabajando en el sistema sanitario?:

3. ¿Profesión: Médica Enfermera

4. ¿Cuál es su puesto de trabajo?:

$$
\text { Atención Primaria ; Hospital ; Ambulatorio especialidades }
$$

5. ¿Ha fumado alguna vez en su vida?

$$
\mathrm{Si} \quad \text { No }
$$

6. ¿Fumo alguna vez durante 6 meses o más diariamente?

$$
\text { Si No }
$$

7. ¿Y ahora cual es su situación?

Fumo diariamente

Fumo ocasionalmentc $(<1$ cigarrillo al día)

No fumo

8. ¿Si fuma a diario ¿que cantidad fuma habitualmente?

$$
\begin{aligned}
& \text { N. }{ }^{\circ} \text { de cigarrillos } \\
& \text { N. }{ }^{\circ} \text { de pipas } \\
& \text { N. }{ }^{\circ} \text { de puros }
\end{aligned}
$$

9. Si fuma, ¿ha intentado seriamente dejar de fumar un mínimo de 24 horas sin fumar?:

$$
\text { Si No }
$$

10. Si fuma, ¿cómo cree que será su hábito tabáquico dentro de 5 años?

Seguramente fumaré a diario

Probablemente a diario

Probablemente no fumaré a diario

Seguramente no fumaré

11. ¿Fuma en presencia de los pacientes?

$$
\text { Si No }
$$

12. ¿Esta permitido fumar en su puesto de trabajo?

$$
\mathrm{Si} \quad \text { No }
$$

13. ¿Se fuma habitualmente en las salas de uso común para el personal (despachos, etc)?

$$
\text { Si No }
$$


14. ¿Existe un lugar específico para fumadores en su lugar de trabajo?

$\mathrm{Si} \quad$ No

15. ¿Piensa que el humo del tabaco perjudica la salud del no fumador?

$\mathrm{Si} \quad$ No

16. ¿Conoce la legislación vigente referida al uso de tabaco en los centros sanitarios?

Si No

17. ¿ Cree que debe permitirse fumar en las salas de esp sra?

$\mathrm{Si} \quad$ No

18. ¿Que valor da a las siguientes razones para no fumar?

- Proteger su salud: Importante No importante

- Aparición de síntomas: Importante No importante

- Presión de sus compañeros/as: Importante No importante

- No molestar a quienes le rodeaban: Importante No importante

- Dar un buen ejemplo: Importante No importante

19. ¿Cuándo cree que debería de aconsejarse a los pacientes que dejen de fumar?

- Afecciones pulmonares: Si No

- Afecciones de las vías aéreas superiores: Si No

- Afecciones cardiacas: Si No

- Ulcera péptica: Si No

- Embarazo: Si No

- Uso de contraceptivos orales: Si No

- Lesiones de la boca o labio: Si No

- A todos/as los/as fumadores/as: $\mathrm{Si}$ No

20. ¿Por favor indique si esta de acuerdo o no con las siguientes afirmaciones:

a) Su conocimiento actual es suficiente para aconsejar a los pacientes que dejen de fumar:

Acuerdo Desacuerdo

b) Debería restringirse la posibilidad de fumar en los lugares públicos cerrados:

Acuerdo Desacuerdo

c) Debería asegurarse el cumplimiento de la legislación vigente sobre el consumo del tabaco en los centros sanitarios:

Acuerdo Desacuerdo

d) Los profesionales sanitarios deberían de recibir formación especifica sobre como ayudar a los pacientes a dejar de fumar:

Acuerdo Desacuerdo

e) Deberia incluirse como hacer prevención del consumo del tabaco en la formación regular de los profesionales sanitarios:

Acuerdo Desacuerdo

f) Debería de prohibirse totalmente la publicidad directa o indirecta sobre el tabaco:

Acuerdo Desacuerdo 
21. ¿Hasta que punto cree que el comportamiento de los/as profesionales sanitarios, como fumadores o no fumadores, puede influir en que los pacientes fumen o no?
a) No tiene influencia
b) Tiene escasa influencia
c) Tiene bastante influencia
d) Tiene mucha influencia 